\title{
Factores que inciden en la elección de carreras STEM en la educación universitaria de El Salvador
}

\author{
Vilma Guadalupe Duke Escobar ${ }^{1}$ \\ José Ovidio Torres Sigüenza² \\ Moisés Ulises García Perdido ${ }^{3}$ \\ Carmen Sofía Toledo Martínez ${ }^{4}$ \\ Docentes investigadores, Facultad de Ciencias de la Salud \\ Universidad Católica de El Salvador, El Salvador
}

Fecha de recepción: 22-09-2020

Fecha de aceptación: 21-01-2021

\section{Resumen}

Según expertos en la rama de educación, la elección de una carrera universitaria marca el rumbo de la vida del individuo; sin embargo, esta tiene bases muy fuertes en la formación académica temprana del estudiante. Contrario a lo que se cree, la toma de decisión no es espontánea al momento de inscribirse en una carrera.

Por ello, con el fin de identificar y entender de una forma integral las causas principales por las cuales los estudiantes optan, o no, por carreras en el área de Ciencia, Tecnología, Ingeniería y Matemática se desarrolló esta investigación, tomando como sujetos de estudio a 540 estudiantes activos, provenientes de seis Instituciones de Educación Superior de El Salvador. Para la recolección de datos, la muestra incluyó tanto estudiantes de carreras de Ciencia, Tecnología, Ingeniería y Matemática como de otras no afines a ellas. Los participantes tomaron un cuestionario de construcción mixta de forma individual.

Al analizar los resultados obtenidos, es posible determinar que, aunque no son exclusivos, dos de los factores que más incidencia tienen en la elección de este tipo de carreras son los socioeconómicos y familiares. En contraposición a lo expuesto por otros autores, el sexo parece no jugar un papel determinante en la elección de carreras en estas áreas, pero sí la formación.

Palabras clave: Educación STEM, educación superior, educación media, elección de carreras, factores de elección, vocación.

\begin{abstract}
According to experts in the field of education, the choice of a university career marks the course of the individual's life; however, it has very strong foundations in the early academic formation of the student. Contrary to popular belief, decision-making is not spontaneous when enrolling in a major.

Therefore, in order to identify and understand in a comprehensive way the main causes why students choose, or not, for careers in the area of Science, Technology, Engineering and Mathematics, this research was developed, taking as study subjects 540 active students from six Higher Education Institutions in El Salvador. For data collection, the sample included both, students from Science, Technology, Engineering and Mathematics careers as well as from others not related to them. Participants took a mixed construction questionnaire individually.

When analyzing the results gathered, it is possible to determine that, although they are not exclusive, two of the factors that most affect the choice of this type of career are socioeconomic and family factors. Contrary to what has been stated by other authors, sex does not seem to play a determining role in the choice of careers in these areas, but training does.
\end{abstract}

Key words: STEM education, higher education, secondary education, career choice, choice factors, vocation.

1. Master of Arts in Curriculum and Instruction; Directora Oficina de Internacionalización; email: vilma.duke@catolica.edu.sv; ORCID: https://orcid.org/0000-0002-5920-3641

2. Master of Arts in TESOL; Docente tiempo completo Facultad de Ciencias y Humanidades; email: jose.torres@catolica.edu.sv; ORCID: https://orcid.org/0000-0002-7775-5116

3. Maestro en Asesoría Educativa; Secretario General; email: moises.garcia@catolica.edu.sv; ORCID: https://orcid.org/00000002-1469-1557

4. Maestra en Asesoría Educativa; Asistente Académica Facultad de Ciencias y Humanidades; email: sofia.toledo@catolica.edu.sv; ORCID: https://orcid.org/0000-0002-8770-1971 


\section{Introducción}

La enseñanza y aprendizaje en las áreas Science, Technology, Engineering and Math (STEM) que por sus siglas en inglés hace referencia a Ciencia, Tecnología, Ingeniería y Matemática, es un modelo educativo que está siendo aplicado durante la última década (Wu-Rorrer, 2017).

Este modelo educativo juega un papel importante, pues a nivel internacional existe una alta demanda de personas capacitadas en estas áreas. En países desarrollados no es una novedad, estos cuentan con programas que además de financiar este tipo de carreras también ayudan a su implementación y mejora constante (Yang, 2017). Hablar de educación STEM es todo un nuevo mundo lleno de tecnología, nuevas competencias como las habilidades de comunicación, creatividad, independencia, entre otras.

Existe una baja demanda a nivel universitario en estas carreras, e internacionalmente la demanda de profesionales en estas áreas aumenta cada día (Finnie y Childs 2018). A nivel nacional, y de acuerdo a las estadísticas ofrecidas por el Ministerio de Educación de El Salvador (2017), de un total de 186,067 estudiantes inscritos, los estudiantes en carreras de tecnología son 35,393 , que equivalen al $19.02 \%$; y los de carreras de ciencias 3,732, que equivalen solamente al $2.01 \%$. Esto significa que el porcentaje de estudiantes en carreras STEM en el 2017 es del 21.03\%.
Lo anterior evidencia que estas áreas aún siguen siendo de baja prioridad y elección por parte de los estudiantes, o que podría relacionarse con diferentes factores como: económicos, sociales, culturales y académicos.

\section{El área de educación STEM}

En años recientes, el interés por promover el estudio de carreras técnicas por parte de la fuerza laboral de países de primer mundo, tales como Estados Unidos y Canadá, se ha visto aumentado significativamente (Stebbins y Goris, 2019). Dada la demanda de personal capacitado en áreas afines a las carreras STEM, tanto Instituciones de Educación Superior como de educación media, se ven cada vez más inclinadas a implementar mallas curriculares que abonen en la promoción de personal capacitado que responda a ésta (Johnson, 2013). Por ello, sería de gran importancia adecuar el currículo educativo de tal forma que sea factible para las instituciones, maestros, estudiantes, padres de familia y el sistema educativo en general, aceptando el reto de mantenerse a la vanguardia de los avances tecnológicos que juegan un papel determinante en el rumbo que toma la sociedad global (Hacioğlu et al., 2016).

\section{Elección de Carreras}

Elegir una carrera universitaria no es una tarea sencilla. Es una decisión que marca el futuro de la persona y se vuelve compleja ya que puede estar influenciada por diversos factores intrínsecos o extrínsecos (Bravo y Vergara, 2018).

5. Dentro del artículo, los autores se referirán a las carreras en el área de Ciencia, Tecnología, Ingeniería y Matemática por sus siglas en inglés, STEM. 
Algunos estudios reflejan que los fenómenos que inciden en el ingreso, permanencia y elección de una carrera universitaria, están relacionados con la percepción subjetiva que tienen los alumnos respecto a sus oportunidades reales, congruentes directamente al origen socioeconómico familiar, al nivel de escolaridad de los padres, el sexo y la edad, como factores que regulan las aspiraciones universitarias (Piñero, 2015).

Así también, el aspecto motivacional es de particular importancia al aspirar a la educación superior y su ausencia supone el no valorar todas las implicaciones personales, laborales y sociales requeridas al momento de elegir una carrera. Sin este aspecto se tiene como efecto directo la apatía hacia la carrera elegida, pero no deseada, traduciéndose en una falta de impulso para involucrarse, mantenerse y concluir con éxito la carrera elegida (Cano, 2008).

Para efectos de esta investigación, a continuación, se describen los aportes de Bravo y Vergara, Piñero, Guzmán, Han y Appelbaum, entre otros, quienes sostienen que, entre los factores de gran influencia respecto a la elección de una carrera universitaria, principalmente en el Área STEM, están el Socio-Económico, Cultural y Formación en Educación Media.

\section{Factores vinculados a la elección de carreras}

\section{Socioeconómico}

Este factor hace referencia, en primer lugar, a la situación económica de la familia y al salario de ésta, en cuanto sea capaz de asumir o no los gastos que implica la formación profesional y con miras a la remuneración que obtendrá el futuro profesional, como medio para mejorar el ingreso económico personal o de la familia (Bravo y Vergara, 2018).

Por consiguiente, quienes desean estudiar una carrera universitaria tienen altas aspiraciones que sostienen su decisión. Los estudiantes que provienen de altos niveles económicos o culturales se inclinarían particularmente a elegir una carrera universitaria que les permita mantener o mejorar su posición social actual, optando por cualquiera de las diversas opciones de carreras. Por otro lado, los que proceden de clases económicas bajas se ven obligados a considerar las opciones posibles, desde las carreras más cortas, con menos demanda de inversión y lógicamente con mayores oportunidades de acceder al mercado laboral posteriormente con más rapidez. Se habla entonces de los costos y beneficios de elegir una carrera en particular (Piñero, 2015).

\section{Cultural}

La elección de la carrera es una decisión que afecta a la familia tanto como ésta influye en ella, dado que el inicio de los hijos a estudios superiores es un tema que aparenta estar reservado para aquellas familias que tienen la capacidad económica de acceder a estos. Poseer un título tiene connotaciones de triunfo, buena remuneración y progreso en la sociedad (Bravo y Vergara, 2018). No obstante, las familias, independientemente de su clase económica, persiguen el objetivo de que sus hijos tengan mejores oportunidades que las que ellos tuvieron (Piñero, 2015). 
Asimismo, es curioso observar que la mayor parte de personas que representan al gremio STEM, tanto a nivel de estudios como profesionales, son hombres, lo cual podría ser entendido desde varias perspectivas (Jiang, Schenke, Sue Eccles, Xu y Warshauer, 2018). En el país, hay sectores de la sociedad, tales como la "Fundación Salvadoreña para el Desarrollo Económico y Social (FUSADES)", que hacen pública su preocupación respecto al bajo número de mujeres desenvolviéndose en áreas profesionales en STEM (Guzmán, 2019). Según Guzmán “La participación de las mujeres en la ciencia es baja tradicionalmente; de todas las personas con título de ingeniería en El Salvador, solo el 7.7\% son mujeres" (2019, párr. 5). Sin embargo, la baja participación del sector femenino en las carreras STEM muestra no ser exclusivo de El Salvador; según Gúzman (2019), el problema es común en un gran número de países latinoamericanos, y aunque no se determina con exactitud las causas, sí se presume que existen razones culturales que intervienen en la toma de decisiones respecto al estudio o no de carreras STEM.

\section{Formación en Educación Media}

Para países cuyos avances en tecnología evolucionan a gran escala, la educación en áreas STEM ha significado un medio efectivo para conformar una fuerza laboral capacitada para afrontar sus retos (Han y Appelbaum, 2018). Esta formación ha demostrado tener un impacto positivo cuando se imparte sistemáticamente desde los inicios de la educación primaria, y es reforzada en la etapa de la educación secundaria (Stebbins y Goris, 2019).

En este sentido la orientación educativa juega un papel clave dentro del contexto educativo al elegir una carrera universitaria. En el nivel de educación media los educandos deberían interiorizar los conocimientos necesarios sobre qué hacer y cómo enfrentar el nivel educativo superior. Cabe resaltar en esta parte que existe un trabajo aislado entre los centros de enseñanza media y los de educación superior (Martínez, Ortega, Sánchez, Cuberos y Garcés, 2016).

Existe, además, evidencia sólida que determina el alto grado de influencia que tiene la formación media en potenciales estudiantes universitarios de áreas STEM en países de Latinoamérica (Amaro, Peña, y Mendoza, 2020). Amaro et al., (2020) ponen en evidencia que en un grupo de estudiantes de ingeniería de Campeche, una de las principales causas del bajo desempeño de ellos se relaciona con la falta de orientación y motivación recibida en su formación media.

Así también, según investigaciones recientes, el aprendizaje en STEM está estrechamente relacionado con el "Deep Learning" (aprendizaje significativo) en cuanto que ambos buscan promover en los estudiantes el desarrollo de estrategias metacognitivas tales como: el pensamiento crítico, la resolución de problemas, entre otros (Mcclure, Clements, Bales, Nichols, Kendall-Taylor y Levine, 2017). Mismas que según expertos son de carácter práctico en la vida cotidiana y, por ende, de mucha relevancia 
en la formación académica. Así, es importante resaltar que serán los docentes los responsables de implementar nuevas estrategias y tecnologías en el área STEM en las escuelas y universidades (Bosch, et al., 2011).

Los elementos definidos anteriormente y los resultados obtenidos, permitieron responder al problema planteado al inicio de la investigación: ¿Qué factores inciden en la elección de carreras STEM por parte de los estudiantes universitarios de El Salvador?

\section{Metodología}

Por su naturaleza, esta investigación es de tipo mixta. Se tomó una muestra no probabilística por conveniencia y fue desarrollada tomando como población de estudio a 540 estudiantes activos, provenientes de seis Instituciones de Educación Superior (IES) ${ }^{6}$ de El Salvador, tal como se muestra en la tabla 1.

La investigación tuvo como finalidad la identificación de los factores que inciden en la elección o no elección de carreras afines a las áreas
STEM. El estudio se desarrolló a partir de datos cualitativos recolectados utilizando la técnica de la encuesta. El instrumento de recolección de datos, cuyo diseño fue de estructuración mixta, constó de 27 ítems, todos ellos organizados sistemáticamente con el propósito de explorar tres variables: factores socio-económicos, culturales y del nivel de Educación Media de los participantes. Para la administración del cuestionario, los estudiantes fueron abordados en sus respectivos salones de clase, en la IES a la cual pertenecían. Aun cuando la administración del cuestionario se realizó en los salones de clase, los participantes tomaron el instrumento de forma individual y personal. Una vez finalizado el proceso de recolección de datos, estos fueron tratados y analizados mediante el uso de la herramienta Microsoft Excel; ello facilitó el análisis estadístico y descriptivo de las variables de forma integral.

Un mismo cuestionario se aplicó a individuos inscritos en carreras afines y no afines a las áreas STEM. Estos se dividieron en grupo A

Tabla 1. Universidades participantes en el estudio

\begin{tabular}{|c|l|c|l|}
\hline No. & \multicolumn{1}{|c|}{ Nombre Universidad } & $\begin{array}{c}\text { Porcentaje } \\
\text { muestra }\end{array}$ & $\begin{array}{c}\text { Zona } \\
\text { geográfica }\end{array}$ \\
\hline 1 & Universidad Gerardo Barrios & $16 \%$ & Oriental \\
\hline 2 & Universidad de Oriente & $16 \%$ & Oriental \\
\hline 3 & Universidad Don Bosco & $20 \%$ & Central \\
\hline 4 & Universidad Centroamericana José Simeón Cañas & $14 \%$ & Central \\
\hline 5 & Universidad de Sonsonate & $16 \%$ & Occidental \\
\hline 6 & Universidad Católica de El Salvador & $18 \%$ & Occidental \\
\hline
\end{tabular}

6. Dentro del documento, los autores se referirán a la Instituciones de Educación Superior mediante sus siglas, IES. 
(estudiantes de carreras afines a STEM) y grupo B (estudiantes de carreras no STEM). Al contar con una muestra representativa de ambas áreas de estudio, fue posible comparar los datos recolectados. Así, se pudo establecer con alto grado de certeza los motivos que jugaron un papel determinante en los participantes al seleccionar la carrera que estudian.

\section{Resultados}

Se presentan los resultados en función de las principales variables de investigación:

La tabla número dos resume los datos generales arrojados por medio del cuestionario. De los 540 estudiantes, 295 son hombres y 245 mujeres, lo que corresponde a un 55\% y $45 \%$ respectivamente, en edades de los 17 a los 38 años.

Respecto a la zona geográfica de procedencia, 416 estudiantes provienen de la zona urbana, 110 estudiantes de la zona rural y 14 no indicaron esta respuesta. Es interesante resaltar que un $67 \%$ de los estudiantes que provienen de la zona urbana se inclinaron por carreras en el área STEM.
Seguidamente, se analizaron tres diferentes variables: socio económica, cultural y formación en educación media. Estas, a su vez, se desglosaron en diferentes indicadores.

\section{Variable socioeconómica}

En relación a esta variable, se analizaron los indicadores: ingresos familiares, gastos académicos y oportunidad laboral en áreas STEM. Uno de los aspectos valorados fue el ingreso familiar con respecto a la carrera que se estudia. Se observa en la figura 1 que los ingresos familiares percibidos por los estudiantes del grupo A son en general mayores.

Otro indicador analizado fue gastos académicos de los estudiantes y la relación de estos con la carrera que estudian. En general, se puede observar que hay una mayor inversión por parte de los estudiantes del grupo A (ver figura 2).

El tercer indicador analizado fue oportunidad laboral en áreas STEM. Se consultó acerca de las áreas de trabajo a futuro. En ambos casos, grupo A y grupo B, contestaron arriba del $75 \%$ que esperan trabajar en áreas afines a sus

Tabla 2. Datos generales

\begin{tabular}{|l|c|c|c|c|}
\hline \multirow{2}{*}{ Datos generales } & \multicolumn{2}{|c|}{ Masculino } & \multicolumn{2}{c|}{ Femenino } \\
\cline { 2 - 5 } & Cantidad & Porcentaje & Cantidad & Porcentaje \\
\hline Grupo A & 229 & $78 \%$ & 113 & $46 \%$ \\
\hline Grupo B & 62 & $21 \%$ & 130 & $53 \%$ \\
\hline No define carrera & 4 & $1 \%$ & 2 & $1 \%$ \\
\hline
\end{tabular}




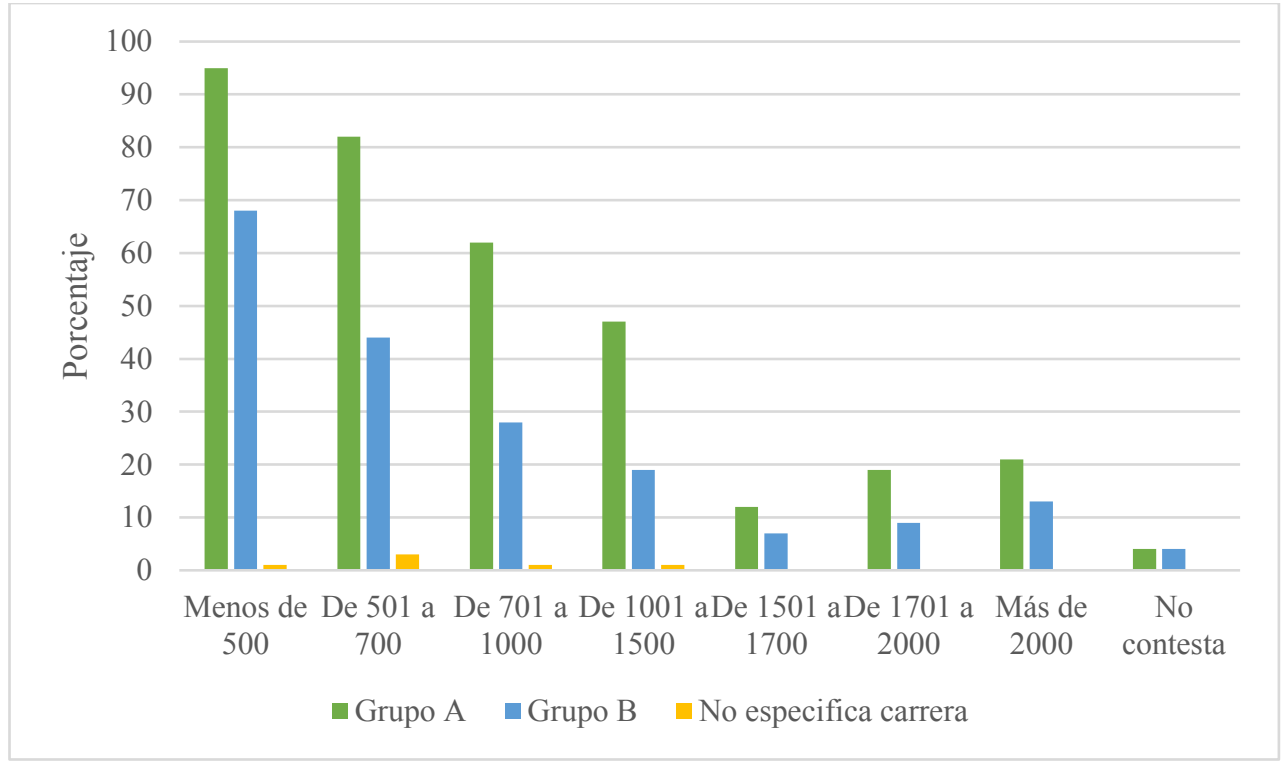

Figura 1. Relación entre salario y carrera.

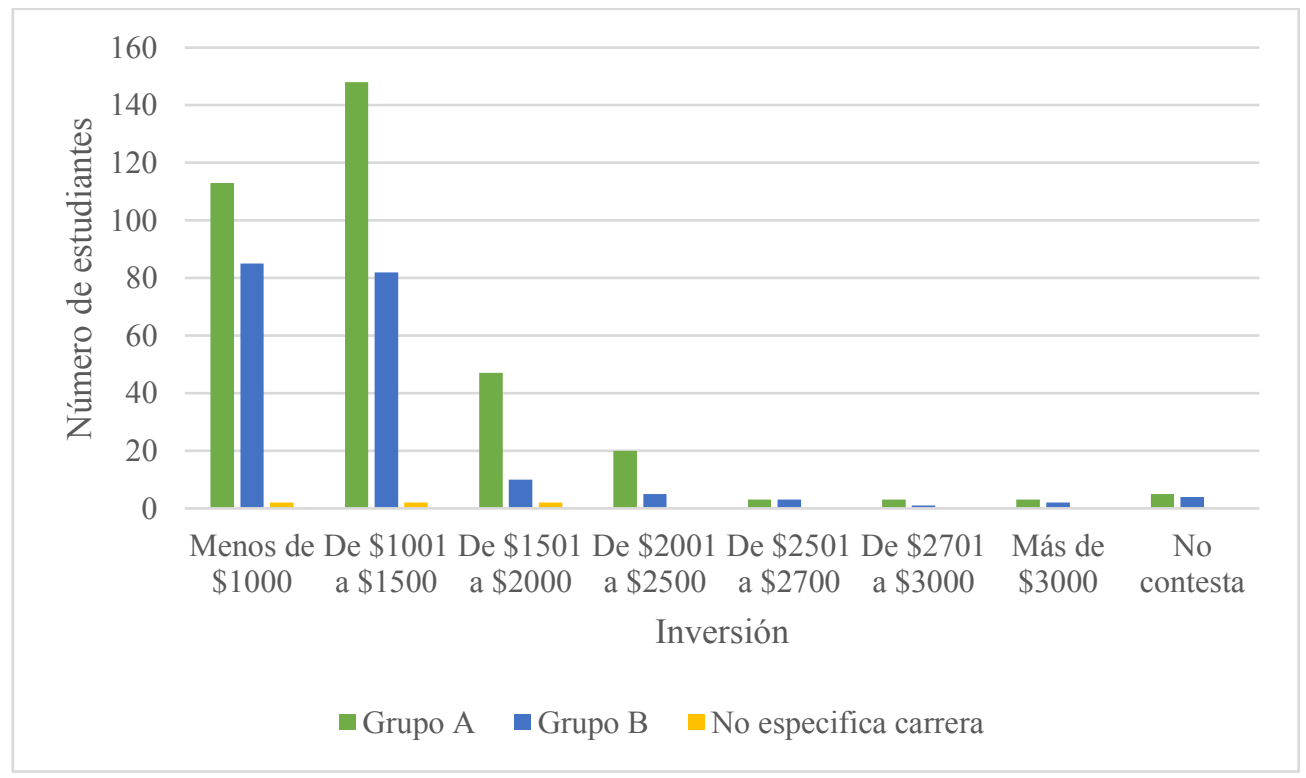

Figura 2. Relación entre tipo de carrera y gastos académicos. 
carreras; y también en ambos casos, más del $50 \%$ contestó que les gustaría tener un negocio propio. Además, se preguntó si tenían conocimiento de empresas o instituciones en las cuales podrán ejercer su profesión al graduarse. De nuevo, en ambos casos, contestaron arriba del $75 \%$ que sí tenían conocimiento. Otra pregunta fue acerca del tipo de empresa en las cuales podrían ejercer, a la que el $68 \%$ contestó que pueden ejercer en empresas privadas; un 5\% respondió que en empresas públicas; el 28\% se limitó a no contestar; y un 2\% contestó que en empresas autónomas o emprendimientos.

Al consultarle a ambos grupos, si conocen empresas en el exterior donde podrían contratarlos al finalizar su carrera, las respuestas fueron bastante homogéneas; del grupo A el 40\% contestó que sí y del grupo B el 42\%. Por último, se consultó si pensaban que la proyección de salario de un profesional en la carrera que eligieron fue un motivo por el cual tomaron la decisión. La figura tres ilustra que del total de estudiantes encuestados el 55\% fue influenciado por la proyección de salario. Sin embargo, si se observan los resultados por áreas, el grupo A tiene un porcentaje mayor que el grupo B, es decir que en estas áreas los estudiantes (63\%) no fueron influenciados por la proyección de salario en la carrera que eligieron.

\section{Variable cultural}

En esta variable se analizaron tres indicadores: prejuicios familiares, antecedentes familiares y apoyo familiar.

Los resultados se presentan de manera general pues no hay una variación significativa al hacer el análisis por áreas. Por tanto, se encontró que en el $30 \%$ de los hogares de donde provienen los jóvenes, sus padres poseen un título profe-

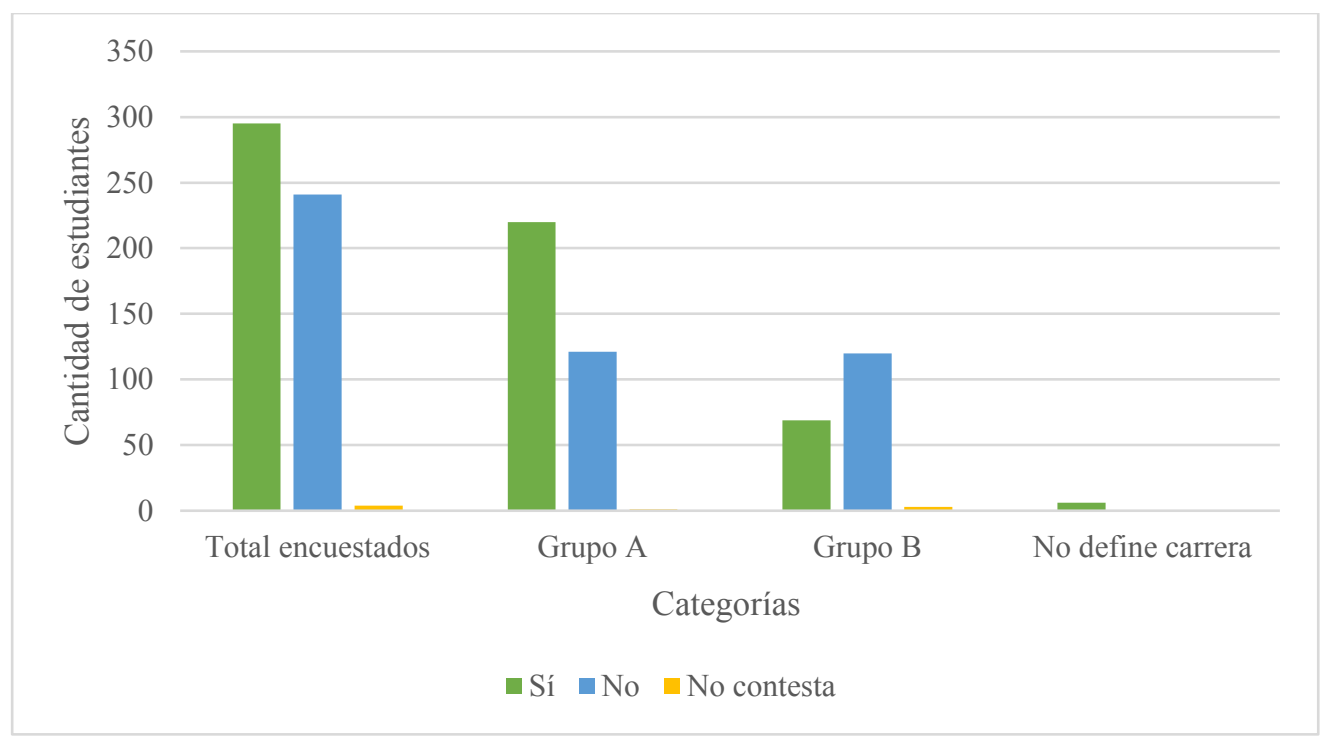

Figura 3. Proyección de salario y elección de carrera. 
sional. Así mismo, se preguntó si en sus familias se escuchó la expresión "hay carreras solo para hombres y otras propias para mujeres"; del total de estudiantes, el $87 \%$ contestó que no ha escuchado tal expresión.

También se indagó respecto al apoyo que reciben los jóvenes por parte de sus familias, aspecto que es muy positivo pues el $79 \%$ de todos los encuestados aseguran haber valorado con sus familias las diferentes opciones ofrecidas por las universidades. De igual forma, en la mayoría de los casos las familias respetaron la decisión de sus hijos en la elección de carreras, pues solo un $26 \%$ de los estudiantes manifiesta que su familia les sugirió otra carrera. Entre las razones se destaca que sus padres consideraban que en una carrera diferente habría mejores posibilidades de empleo, además, por influencia familiar, prestigio de la carrera, tradición, factores económicos, y potencial del joven.

La última pregunta de esta variable cuestionó a los estudiantes respecto a su economía familiar y la decisión de estudiar una carrera; solo el $26 \%$ de todos los encuestados contestó que la situación económica influyó en la elección de su carrera.

\section{Variable formación en educación media}

En esta variable los indicadores fueron datos académicos, metodología utilizada, rol del docente y temor a ciertas áreas del conocimiento. Respecto a los datos académicos, del total de estudiantes encuestados el $68 \%$ proviene de ba- chillerato general, el restante $32 \%$ proviene de un bachillerato técnico. Independiente del tipo de bachillerato de procedencia, arriba del 60\% de los estudiantes se inclinaron por carreras en las áreas STEM. Así también, el 49\% estudió su bachillerato en instituciones públicas, mientras que el $51 \%$ lo hizo en instituciones privadas. El porcentaje de ellos que optó por carreras STEM o no STEM, se muestra en la figura número cuatro. Se puede observar que un alto porcentaje de estudiantes provenientes de instituciones privadas $(69 \%)$ optaron por carreras STEM (ver figura 4).

En referencia a la metodología utilizada por los docentes, se preguntó a los estudiantes si sus docentes variaban las mismas durante el bachillerato, el 77\% del total de estudiantes encuestados respondió que sí. Además, se presentó un listado de algunas de las metodologías que más se utilizan para que pudieran seleccionar cuáles de ellas habían utilizado sus docentes. En la figura número cinco se puede observar que no se presentan diferencias entre el total de estudiantes y las diferentes categorías (ver figura 5).

Así mismo, se preguntó a los estudiantes si consideraban que la metodología utilizada por sus docentes había facilitado su aprendizaje, a lo que el $84 \%$ de los encuestados opinó que sí. Otro aspecto muy importante fue conocer si los aprendizajes adquiridos en el bachillerato les son útiles en la universidad, aquí el 84\% respondió que sí. Sin embargo, es importante mencionar que el $15 \%$ de estudiantes que con- 


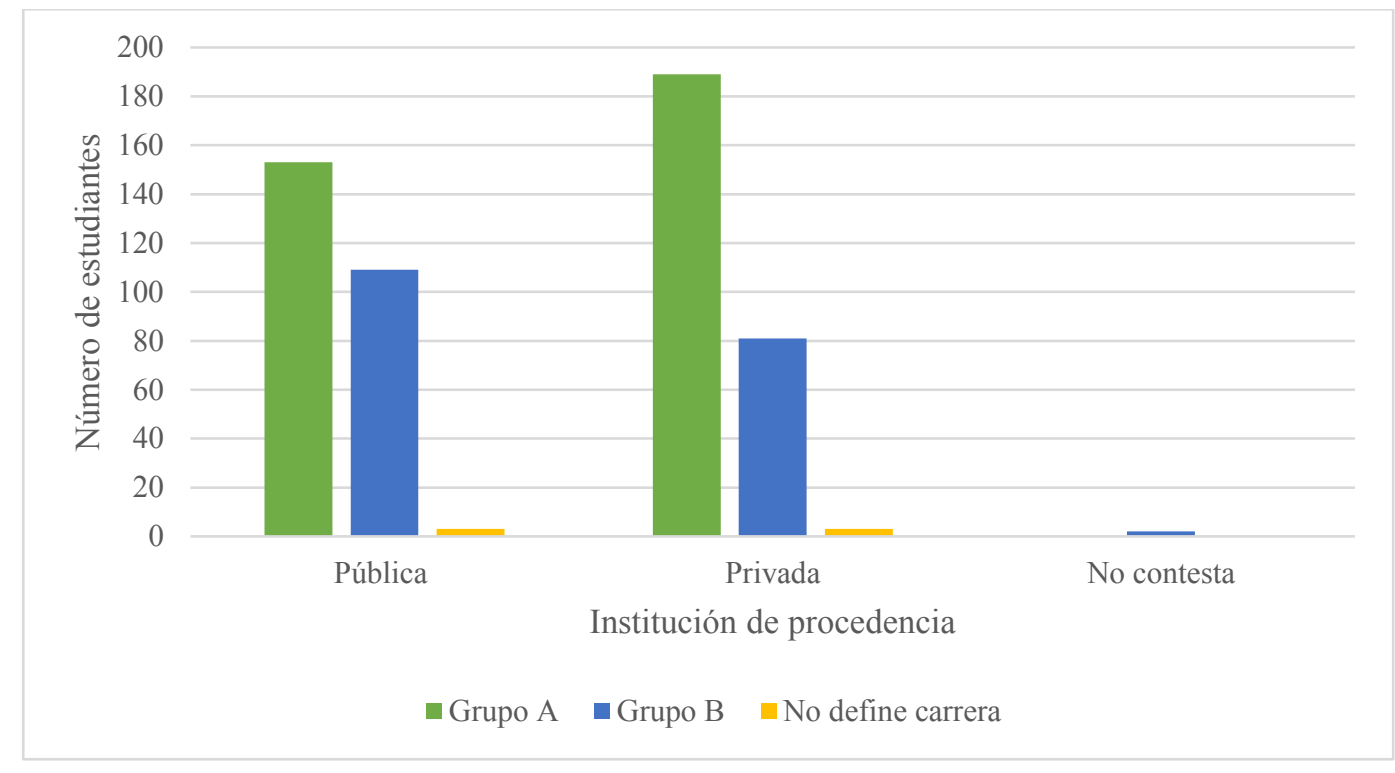

Figura 4. Relación entre la elección de carrera y tipo de bachillerato de procedencia.

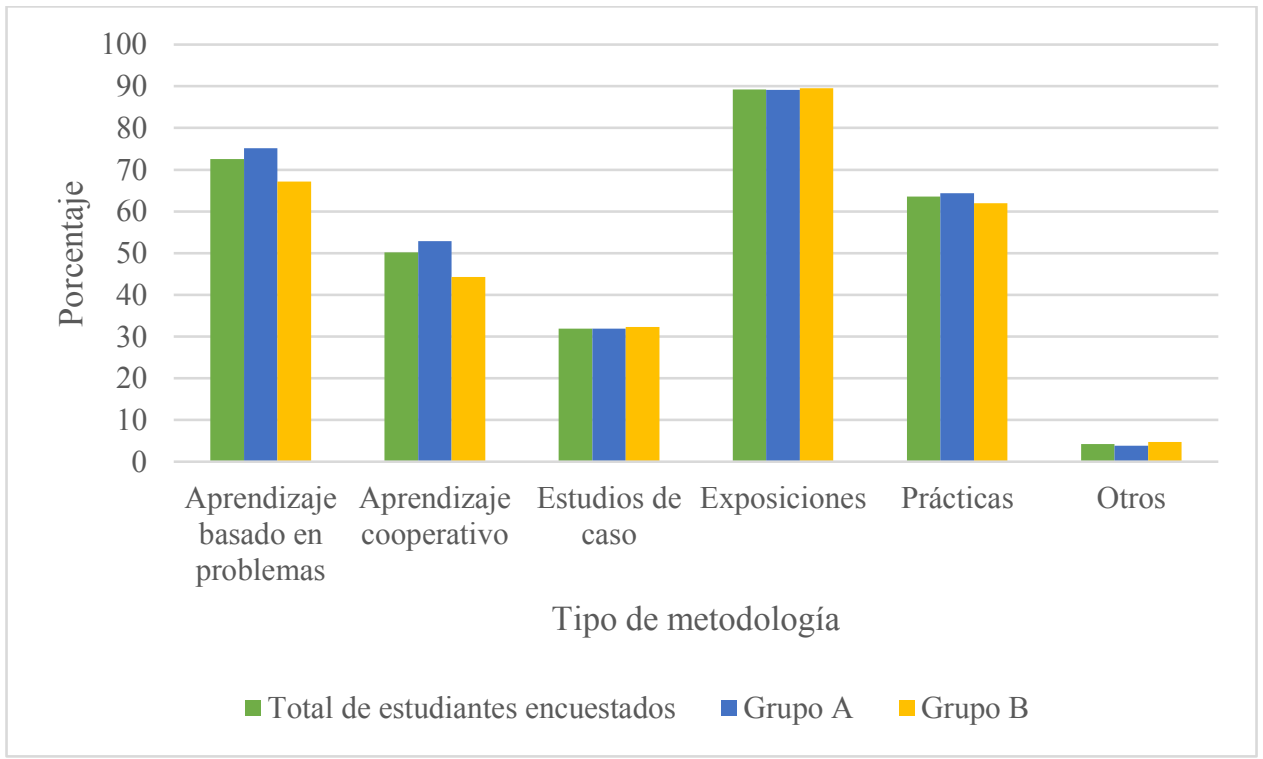

Figura 5. Metodologías utilizadas por los docentes en bachillerato. 
sidera que los aprendizajes no les son útiles exponen razones como: falta de profundidad en el contenido, falta de afinidad en las áreas de estudio del bachillerato y la universidad, la metodología, falta de tiempo para terminar su plan de estudios, etc.

Por otra parte, se preguntó a los estudiantes si sus docentes se tomaron alguna vez el tiempo para animarlos a continuar sus estudios universitarios, el 89\% contestó que sí, la figura número seis muestra las asignaturas que impartieron estos docentes.

Otro aspecto que se investigó fue la relación entre la elección de carrera y si esta posee o no componentes numéricos. Se preguntó a los estudiantes si le resultaban fáciles las materias numéricas. A nivel general, se puede observar que a la mayoría de estudiantes a veces les resulta fácil estas materias; sin embargo, se puede observar un ligero cambio al hacer las comparaciones de los porcentajes entre el grupo $\mathrm{A} y$ grupo B, pues se observa un mayor porcentaje de estudiantes del grupo A qué opinan que se les hace fácil las materias numéricas (ver figura 7).

Así también, se preguntó a los estudiantes respecto al sentimiento o emoción que les generaban las materias numéricas. En el grupo A existe un poco más de agrado por estas materias que en el grupo B (ver figura 8).

Y, finalmente, se consultó acerca de su habilidad en las áreas de Matemática, Ciencias Naturales y Tecnología. Se puede observar la figura nueve que la mayoría de estudiantes, independientemente del área a la que pertenezcan sus

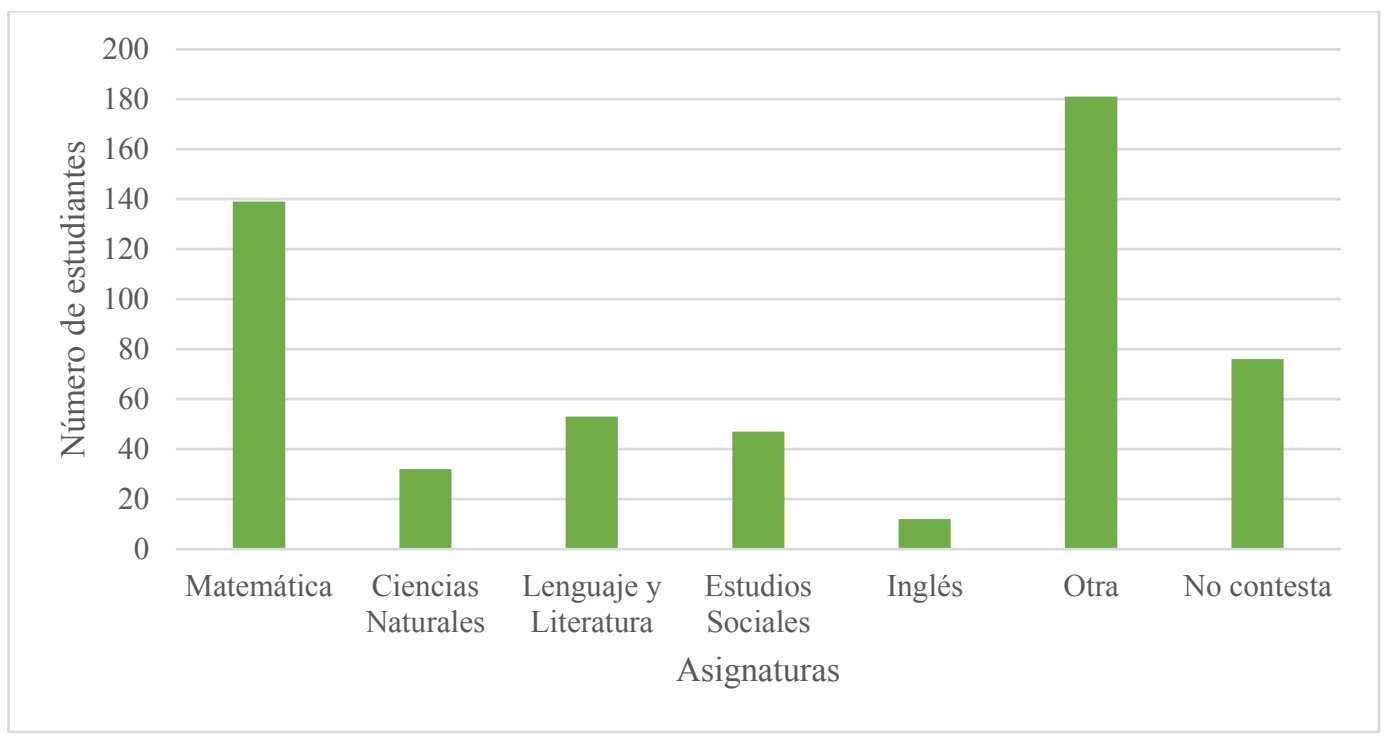

Figura 6. Asignaturas de los docentes que cumplieron un rol motivador. 


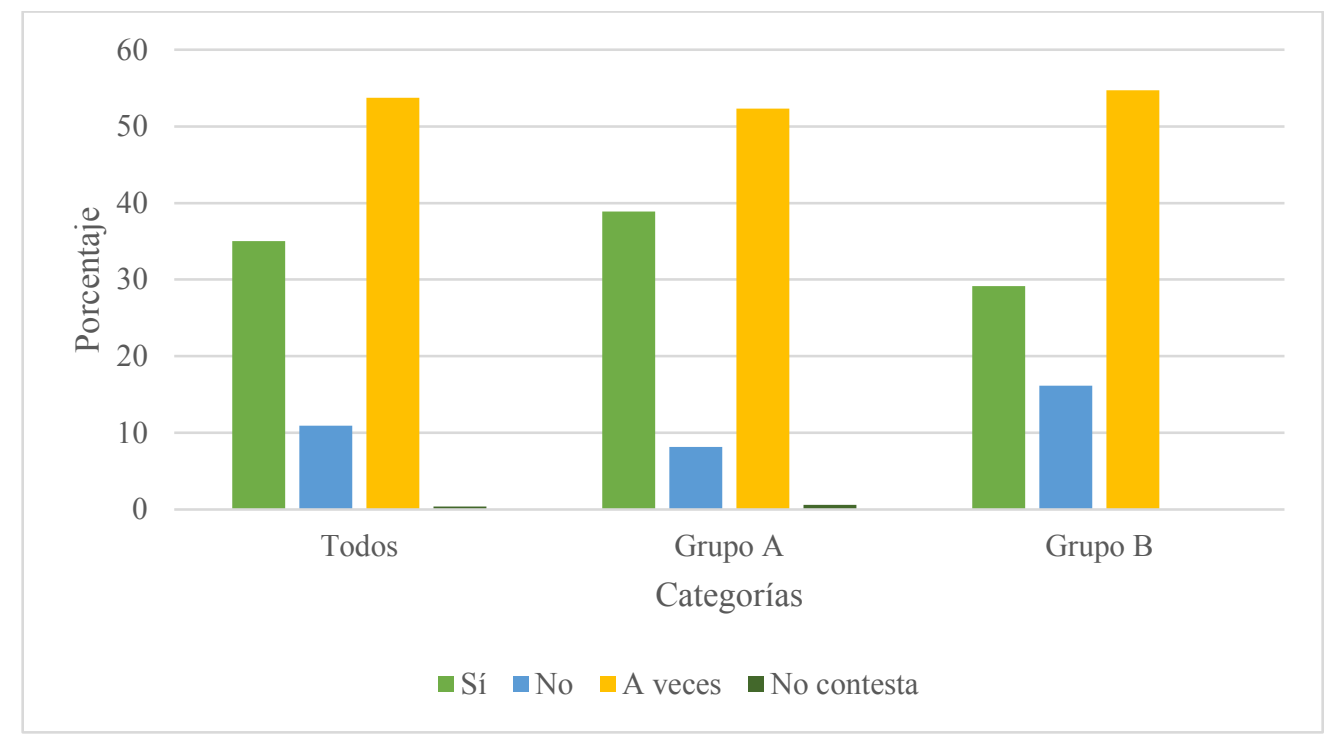

Figura 7. Facilidad de los estudiantes para las materias numéricas.

carreras, consideran sus habilidades en las áreas descritas con un dominio medio; sin embargo, también es posible observar que los estudiantes del grupo A, sobresalen en los dominios altos en las tres áreas. Así mismo, se observa que los estudiantes del grupo B tienden a considerar sus dominios en estas áreas como bajos.

\section{Discusión}

Con base a los resultados obtenidos, existe evidencia suficiente para determinar que el factor económico juega un papel muy importante en cuanto a la elección de carreras afines y no afines a las áreas STEM en El Salvador. En primer lugar, tanto por la inversión en materiales como por la inversión en cuotas de matrícula y mensualidad, el estudio de una carrera STEM requiere de un poder adquisitivo mayor por parte del estudiante o quien financie su carrera. Por otra parte, aun cuando la inversión para estudiar una carrera del área STEM es mayor, se evidencia que ello es compensado por la proyección salarial laboral de los estudiantes a futuro. Los estudiantes son conscientes de ello y por ende invierten en algo cuyos frutos serán certeros al graduarse. Caso contrario ocurre con los estudiantes en carreras no afines a las áreas STEM.

En cuanto al factor cultural se pudo observar que el apoyo familiar respecto a la educación superior de los hijos no se limita únicamente a sufragar el costo de ésta, sino el considerar las posibilidades de ofertas académicas junto a ellos y el respeto por la vocación personal de estos. Así también, es importante resaltar que el género no condiciona la elección o no de carreras STEM, ni el acceder a la educación superior, inclusive cuando algunos padres sugirieron la elección de otra carrera distinta a la actual, sin presentar injerencia directa con el prejuicio de ser hombre o mujer. 


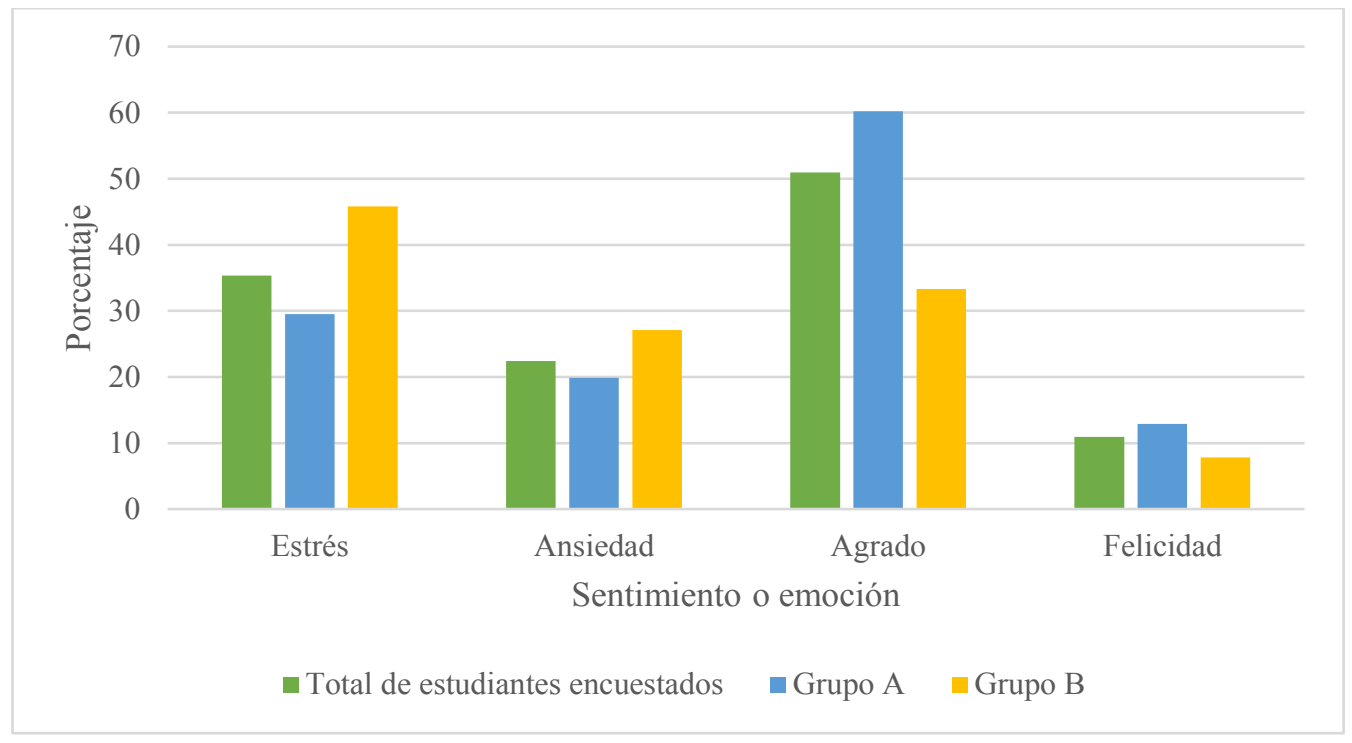

Figura 8. Sentimiento o emoción que generan las materias numéricas.

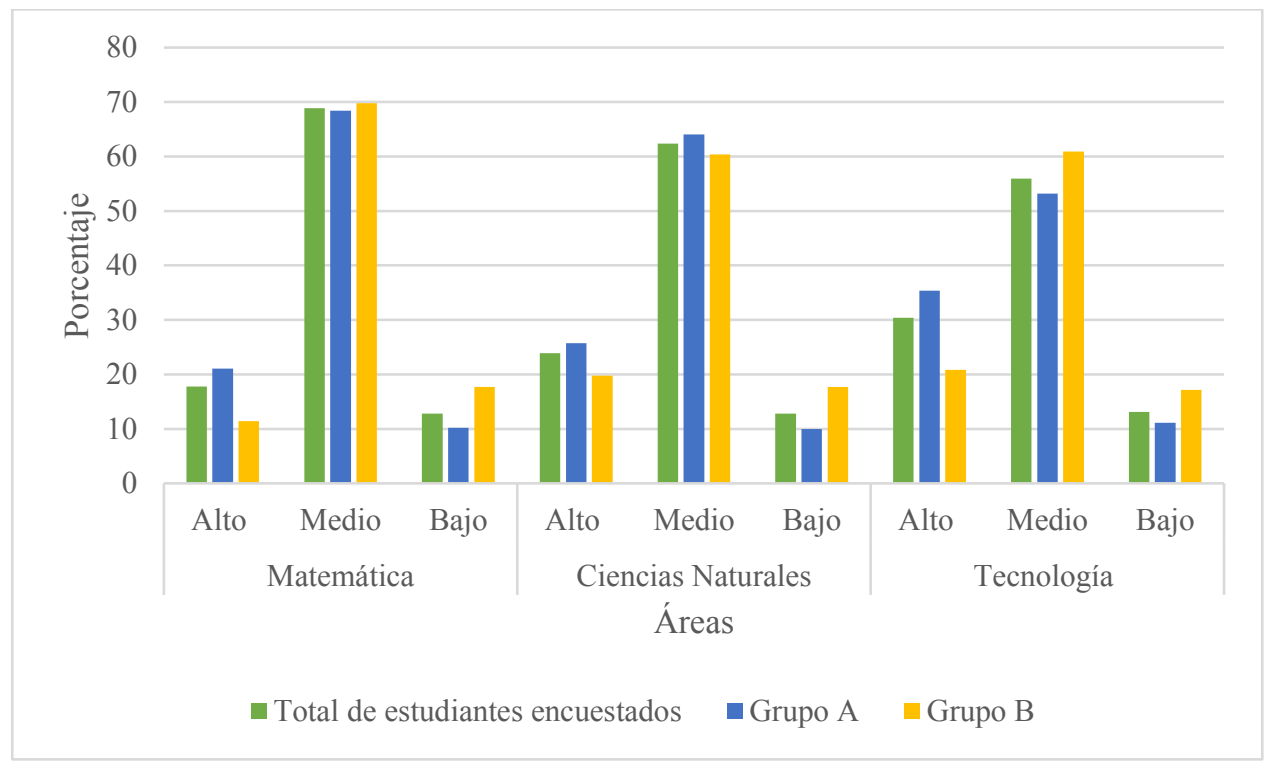

Figura 9. Habilidad de los estudiantes en las áreas STEM. 
Respecto al área de formación en educación media, un aspecto positivo encontrado a la luz de los resultados está relacionado con la calidad de la enseñanza como base de los aprendizajes posteriores en educación superior; además, el rol del docente, en cuanto orienta y motiva a los estudiantes a continuar con su formación universitaria. Sin embargo, también se encontró que los estudiantes consideran que los contenidos cubiertos durante su bachillerato no se tocan a profundidad, por lo que se recomienda una investigación que busque establecer la relación entre el currículo y las demandas actuales de formación. De igual forma, se sugiere instalar mecanismos en educación media que fortalezcan la orientación vocacional.

Así también, los resultados de esta investigación arrojan que un $63 \%$ de los estudiantes encuestados optaron por carreras en el área STEM, a pesar de que en estadísticas de 2017 se ve un porcentaje reducido de estudiantes que optan por estas, alrededor de un $20 \%$. Cabe resaltar que la muestra tomada para esta investigación solo partió de instituciones privadas. Se recomienda para futuras investigaciones incorporar las instituciones públicas de educación superior.

\section{Referencias}

Amaro, L., Peña, J., y Mendoza, C. (2020). Desarrollo de habilidades STEM en media superior como mecanismo para impulsar la continuidad en educación superior: Caso programa Bases de Ingeniería. Revista Iberoamericana para la Investigación y el Desarrollo Educativo, 10 (20). https://doi.org/10.23913/ride.v10i20.614

Bosch, H. E., Di Blasi, M. A., Pelem, M. E., Bergero, M. S., Carvajal, L., y Geromini, N. S. (2011). Nuevo paradigma pedagógico para enseñanza de ciencias y matemática. Avances en ciencias e ingeniería, 2(3), 131-140. https://dialnet.unirioja.es/servlet/articulo?codigo=3752199

Bravo, G., Vergara, M. (2018). Factores que determinan la elección de carrera profesional: en estudiantes de undécimo grado de colegios públicos y privados de Barrancabermeja. Revista Psicoespacios, 12(20), 47-58. https://doi.org/10.25057/issn.2145-2776

Cano, M. (2008). Motivación y elección de carrera. Revista Mexicana de Orientación Educativa, 5(13), 6-9. http://pepsic.bvsalud.org/scielo.php?script=sci_arttext\&pi$\mathrm{d}=\mathrm{S} 1665-75272008000100003 \& \operatorname{lng}=$ pt\&tlng=es.

Finnie, R., y Childs, S. (2018). Who goes into STEM disciplines? evidence from the youth in transition Survey. Canadian Public Policy, 44(S1), S43-S55. https://doi.org/10.3138/cpp.2017-077 
Guzmán, J. (2019, 19 de junio). Solo 8 de cada 100 ingenieros en El Salvador son mujeres. El Diario de Hoy. https://www.elsalvador.com/eldiariodehoy/solo-8-de-cada-100-ingenierosen-el-salvador-son-mujeres/614173/2019

Hacioğlu, Y., Yamak, H., y Kavak, N. (2016). Pre-service science teachers' cognitive structures regarding science, technology, engineering, mathematics (STEM) and science education. Journal of Turkish Science Education, 13(special), 88-102. https://doi:10.12973/tused.10173a

Han, X., y Appelbaum, R. P. (2018). China's science, technology, engineering, and mathematics (STEM) research environment: A snapshot. PloS one, 13(4). https://doi.org/10.1371/journal. pone. 0195347

Jiang, S., Schenke, K., Eccles, J. S., Xu, D., y Warschauer, M. (2018). Cross-national comparison of gender differences in the enrollment in and completion of science, technology, engineering, and mathematics. Massive Open Online Courses. PloS one, 13(9). https://doi.org/10.1371/ journal.pone. 0202463

Johnson, C. (2013). Conceptualizing integrated STEM education. School Science and Mathematics, 113(8), 367-368. https://doi.org/10.1111/ssm.12043

León, T. y Rodríguez, R. (2008). El efecto de la orientación vocacional en la elección de carrera. Revista Mexicana de Orientación Educativa, 5(13), 10-16. http://pepsic.bvsalud.org/scielo. php?script=sci_arttext\&pid=S1665-75272008000100004\&lng=pt\&tlng=es

Martínez-Martínez, A., Zurita-Ortega, F., Castro-Sánchez, M., Chacón-Cuberos, R., Hinojo-Lucena, M. A., y Espejo-Garcés, T. (2016). Choosing Higher Education for Senior High School and Upper Education Students. Revista Electrónica Educare, 20(1), 304-321. http://dx.doi. org/10.15359/ree.20-1.14

McClure, E., Guernsey, L., Clements, D., Bales, S., Nichols, J., Kendall-Taylor, N., \& Levine, M. (2017). How to integrate STEM into early childhood education. Science and Children, 55(2), 8. http://dx.doi.org/10.2505/4/sc17_055_02_8

Ministerio de Educación. (2017). Resultados de la información estadística de Instituciones de Educación Superior 2017. Editorial Panamericana.

Pérez Tyteca, P., Castro Martínez, E., Segovia Álex, I., Castro Martínez, E., Fernández García, F. R., y Cano García, F. (2009). El papel de la ansiedad matemática en el paso de la educación secundaria a la educación universitaria. PNA: Revista de investigación en Didáctica de la Matemática, 4 (1), 23-35. https://doi.org/10.30827/pna.v4i1.6171 
Ramírez, S. L. (2015). Factores asociados a la selección de carrera: una aproximación desde la Teoría de la Acción Racional. CPU-e, Revista de Investigación Educativa, (20), 72-99.

Stebbins, M., y Goris, T. (2019). Evaluating STEM Education in the US Secondary Schools: Pros and Cons of the «Project Lead the Way» Platform. International Journal of Engineering Pedagogy, 9(1), 50-56. https://doi.org/10.3991/ijep.v9i1.9277

Torres, G. B., y Tamayo, M. A. V. (2018). Factores que determinan la elección de carrera profesional: en estudiantes de undécimo grado de colegios públicos y privados de Barrancabermeja. Psicoespacios, 12(20), 35-48.

Wu-Rorrer, R. (2017). Filling the gap: integrating STEM into career and technical education middle school programs. Technology and Engineering Teacher, 77(2), 8-15. https://www.iteea. org/File.aspx?id=137382\&v=4dc600db

Yang, C.-L. (2017). Self-Flipped Learning \& Teaching for STEM in Higher Education. European Society for Socially Embedded Technologies. https://dl.eusset.eu/handle/20.500.12015/2947 